\title{
Optimal Aeroassisted Intercept Trajectories at Hyperbolic Speeds
}

\author{
Elmer G. Gilbert, ${ }^{*}$ Robert M. Howe, $\dagger$ Ping Lu, $\ddagger$ and Nguyen X. Vinh* \\ University of Michigan, Ann Arbor, Michigan 48109
}

\begin{abstract}
This paper considers the optimization of Earth-based multistage rocket interceptors with very short flight times and long ranges. The objective is the minimization of the launch mass as a function of interceptor design variables such as stage size, engine burn times, and the angle-of-attack program. Because of the demanding target conditions, the payload reaches hyperbolic speeds and the centrifugal forces greatly exceeds the gravity force. The minimization of launch mass shows that the needed downforce on the payload is best provided by negative aerodynamic lift. The description of such negative-lift, aeroassisted optimal trajectories is a principal goal of the paper. Topics treated include the following: a model for the multistage interceptor, the formulation of the optimization problem, the mathematical derivation of a universal curve that provides a simple and accurate model for negative-lift segments of the optimal trajectories, and effective procedures for efficient numerical optimization. Results of solution studies are reported. For flight times of $6 \mathrm{~min}$ and a range of about $\mathbf{3 0 0 0}$ miles, a five-stage interceptor requires a mass ratio of several thousand. The dependence of the optimal mass ratio on key design and target parameters is described.
\end{abstract}

\section{Nomenclature}

$\begin{array}{ll}A & =\text { dimensionless thrust in } g=T /\left(m_{0} g_{0}\right) \\ C_{L}, C_{D} & =\text { lift and drag coefficients } \\ D & =\text { dimensionless drag force }=\frac{1}{2} \rho v^{2} S C_{D} /\left(m_{0} g_{0}\right) \\ g_{0} & =\text { gravitational acceleration at } r_{0} \\ H & =\text { dimensionless altitude }=h / r_{0} \\ h & =\text { altitude }=r-r_{0} \\ h_{S} & =\text { scale height of exponential } \\ & \text { atmosphere }=7.16 \mathrm{~km} \\ I_{\mathrm{sp}} & =\text { dimensionless specific impulse }=i_{\mathrm{sp}} /\left(r_{0} / g_{0}\right)^{\frac{1}{2}} \\ i_{\mathrm{sp}} & =\text { specific impulse } \\ L & =\text { dimensionless lift force }=\frac{1}{2} \rho v^{2} S C_{L} /\left(m_{0} g_{0}\right) \\ M & =\text { dimensionless mass }=m / m_{0} \\ m & =\text { vehicle mass } \\ m_{0} & =\text { reference mass } \\ N & =\text { number of stages } \\ R & =\text { dimensionless distance }=r / r_{0} \\ r & =\text { distance of vehicle from center of Earth } \\ r_{0} & =\text { reference distance } \\ S & =\text { cross-sectional reference area } \\ T & =\text { engine thrust } \\ t & =\text { real time } \\ V & =\text { dimensionless speed }=v /\left(g_{0} r_{0}\right)^{\frac{1}{2}} \\ v & =\text { vehicle speed } \\ x & =\text { dimensionless time for universal curve } \\ \alpha & =\text { angle of attack } \\ \gamma & =\text { flight-path angle with respect to local horizontal } \\ \eta & =\text { dimensionless force coefficient }=\rho_{0} r_{0} S / m_{0} \\ \theta & =\text { polar angle of vehicle trajectory } \\ \lambda & =\text { fineness ratio }=\text { stage length/stage base diameter } \\ \mu & =\text { dimensionless inverse scale height }=r_{0} / h_{S} \\ & \end{array}$

Received July 24, 1989; presented as Paper 89-3444 at AIAA Guidance, Navigation and Control Conference, Boston, MA, Aug. 14-16, 1989; revision received Nov. 1, 1989. Copyright (C) 1989 by the American Institute of Aeronautics and Astronautics, Inc. All rights reserved.

*Professor, Department of Aerospace Engineering.

$\dagger$ Professor, Department of Aerospace Engineering. Associate Fellow AIAA

¥Assistant Research Scientist, Department of Aerospace Engineering; currently, Assistant Professor, Department of Aerospace Engineering, Iowa State University, Ames, IA.

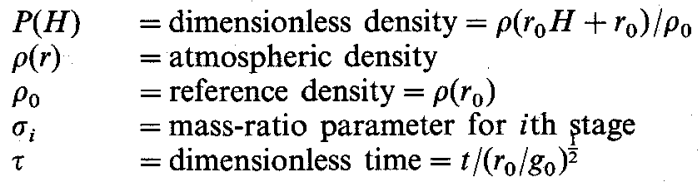

\section{Introduction}

T HE utilization of satellite-based missiles for intercepting intercontinental ballistic missiles (ICBMs) during their ascent constitutes one of the major efforts in the U.S. Strategic Defense Initiative. Here, an alternative approach is considered: the use of high-performance, multistage, Earth-based interceptors. The very short flight times and long ranges require hyperbolic speeds and a high ratio of takeoff mass to payload mass. A natural objective is the minimization of the mass ratio with respect to interceptor design variables such as stage size, engine burn times, coasting times between stages, and the angle-of-attack program. Using techniques described in this paper, numerical solutions of such minimization problems have been obtained. In addition to giving information on how target parameters affect the optimum launch mass, the solutions show that negative aerodynamic lift plays a crucial role in the midcourse portion of the optimal trajectories. The general physical basis for this key result is easy to understand: The hyperbolic speed generates a centrifugal force far in excess of the gravity force, and the required downforce is generated more efficiently by negative aerodynamic lift than by engine thrust. The primary objectives of this paper are threefold: 1) to summarize the models and methods by which the optimal aeroassisted trajectories are computed; 2) to clarify by analysis the detailed nature of the aeroassisted portion of the optimal trajectories; and 3) to give some insight into how design and target parameters affect the optimal launch mass.

The presentation begins in Sec. II with the details of the mathematical model for the multistage interceptor. Physically reasonable simplifying assumptions are introduced to keep the complexity of the model within acceptable bounds. The minimum launch mass problem is formulated in Sec. III. To emphasize the importance of the negative aerodynamic lift, three examples of optimal trajectories are given. They correspond to different assumptions on the trajectory of the payload stage: extra-atmospheric flight, atmospheric flight with zero aerodynamic lift, and negative-lift aeroassisted flight. 
With respect to launch mass the aeroassisted flight offers a clear advantage.

Over a wide range of target conditions it has been observed that the optimal aeroassisted trajectories share a common feature. The atmospheric segments of the payload trajectories follow closely a speed-altitude relationship that we call the universal curve. The theory of this universal curve is developed in Sec. IV. It provides simple formulas that describe accurately the aeroassisted motion of the payload.

Section $\mathrm{V}$ reviews techniques used in the computation of the optimal trajectories. The minimum launch mass problem is a nonlinear, multistage, optimal control problem with parameters and constraints. The effect of its inherent complexity on the computations is eased by using a direct method in which the angle-of-attack program is approximated by a finite-dimensional functional representation. The resulting finite-dimensional optimization problem is solved efficiently by the application of an augmented-Lagrangian, quasi-Newton algorithm. Important practical aspects of the overall procedure are discussed. For example, it is shown how the angle-of-attack program can be parameterized indirectly through a direct parameterization of the flight-path angle. This step is crucial in obtaining a numerically well-conditioned optimization problem.

Many optimal trajectories have been computed. Some of the results are summarized in Sec. VI. They show the effect on launch mass of such parameters as the time of flight, the intercept altitude, the number of stages, and the specific impulse of the engines. For five- and six-stage interceptors mass ratios on the order of several thousand are necessary. Short flight times and low intercept altitudes increase appreciably the launch mass.

\section{Model for the Multistage Interceptor}

To obtain the equations of motion, it is assumed that all stages of the interceptor are modeled as a point mass moving in a plane that contains the center of a spherical, nonrotating Earth with an inverse-square gravitational field. Choosing state variables $r, \theta, v, \gamma$, and $m$ and writing the resulting equations ${ }^{1}$ in dimensionless form then gives

$$
\begin{aligned}
\frac{\mathrm{d} R}{\mathrm{~d} \tau} & =V \sin \gamma \\
\frac{\mathrm{d} \theta}{\mathrm{d} \tau} & =V \frac{\cos \gamma}{R} \\
\frac{\mathrm{d} \gamma}{\mathrm{d} \tau} & =\frac{1}{V}\left[\frac{A \sin \alpha+L}{M}+\left(\frac{V^{2}}{R}-\frac{1}{R^{2}}\right) \cos \gamma\right] \\
\frac{\mathrm{d} V}{\mathrm{~d} \tau} & =\frac{A \cos \alpha-D}{M}-\frac{\sin \gamma}{R^{2}} \\
\frac{\mathrm{d} M}{\mathrm{~d} \tau} & =\frac{-A}{I_{\mathrm{sp}}}
\end{aligned}
$$

These equations are well scaled when $r_{0} \cong$ Earth radius and $m_{0} \cong$ nominal mass of vehicle. Note that $V=1$ corresponds to the circular orbital speed at $r_{0}$, and $\tau=2 \pi$ corresponds to the period of a circular orbit at $r_{0}$.

The dimensionless lift and drag forces are given in terms of $H$ and $V$ by

$$
\begin{aligned}
& L=\frac{1}{2} \eta P(H) V^{2} C_{L} \\
& D=\frac{1}{2} \eta P(H) V^{2} C_{D}
\end{aligned}
$$

For the results reported later in this paper an exponential variation of density with altitude has been used. This approximation simplifies the computations and produces almost the same optimal trajectories as more complex atmospheric mod- els. For the exponential variation,

$$
P(H)=e^{-\mu H}
$$

Lift and drag coefficients are modeled by the formulas ${ }^{2}$

$$
\begin{aligned}
& C_{L}=C_{N} \cos \alpha-C_{A} \sin \alpha \\
& C_{D}=C_{N} \sin \alpha+C_{A} \cos \alpha \\
& C_{A}=0.13 \\
& C_{L}=\sin 2 \alpha \cos \frac{1}{2} \alpha+5(\pi)^{-1} \lambda \sin \alpha|\sin \alpha|
\end{aligned}
$$

In addition to choosing an angle-of-attack program, $\alpha(\tau)$, in Eq. (1), it is necessary to specify the thrust program and describe parametrically the physical characteristics of the stages. Our model of the thrust program is simple and is consistent with the requirements of solid-fuel engines. For the $i$ th stage it consists of an initial coasting period, $\tau_{i}^{C}$, where $A=0$, and a single thrusting period, $\tau_{i}^{B}$, where $A=$ const $>0$. The first stage has no coasting period $\left(\tau_{1}^{C}=0\right)$, and there are $N$ powered stages. The final payload stage is unpowered but may generate a controlled aerodynamic lift. Its coasting time from burnout of the $N$ th stage to target interception is denoted by $\tau_{N+1}^{C}$.

The stage masses are modeled as follows. For the $i$ th stage let $M_{i}^{P}, M_{i}^{F}$, and $M_{i}^{S}$ denote the masses of the payload, fuel, and structure (together with the engine and other jettisoned components), respectively. Then

$$
\begin{aligned}
& M_{i}^{P}=M_{i+1}^{P}+M_{i+1}^{F}+M_{i+1}^{S}, \quad i=0, \ldots, N-1 \\
& M_{0}^{P}=\text { launch mass of entire vehicle } \\
& M_{N}^{P}=\text { mass of final payload }
\end{aligned}
$$

Let

$$
\sigma_{i+1} M_{i}^{P}=M_{i+1}^{P}, \quad i=0, \ldots, N-1
$$

Here, $0<\sigma_{i}<1$ is a stage mass ratio parameter. Once $\sigma_{1}, \ldots, \sigma_{N}$ and $M_{N}^{P}$ are specified, the individual stage payload masses $M_{0}^{P}, \ldots, M_{N-1}^{P}$ are known. To avoid the need for additional stage size parameters, a final simplifying assumption is made:

$$
M_{i}^{S}=0.1 M_{i}^{F}, \quad i=1, \ldots, N
$$

Then from Eqs. (5) it follows that

$$
M_{i}^{F}=(1.1)^{-1}\left(M_{i-1}^{P}-M_{i}^{P}\right), \quad i=1, \ldots, N
$$

Although Eq. (7) neglects many details of the structural design, it is representative of attainable structural efficiencies. Once the fuel masses are determined, the thrust levels $A_{i}$ can be computed. They are proportional to $M_{i}^{F} / \tau_{i}^{B}$.

The masses of stages also affect, through their reference areas, the aerodynamic forces. Let $S_{i}, d_{i}$, and $\lambda_{i}$ be, respectively, the base area, base diameter, and fineness ratio of the $i$ th stage including its payload. Assume that the mass density of each stage prior to the ignition of stage burning is the same. Then, $m_{i-1}^{P}=($ const $) d_{i}^{3} \lambda_{i}$. Since $S_{i}$ is proportional to $d_{i}^{2}$, this gives

$$
\begin{aligned}
S_{i} & =C_{S}\left(m_{i-1}^{P} \lambda_{i}^{-1}\right)^{2 / 3} \\
& =C_{S} m_{0}^{2 / 3}\left(M_{i-1}^{P} \lambda_{i}^{-1}\right)^{2 / 3}, \quad i=1, \ldots, N+1
\end{aligned}
$$

We have chosen $C_{S}$ to make Eq. (9) conform closely with the corresponding relation for the Minuteman I vehicle. In our computations $\lambda_{i}=10$ for $i=1, \ldots, N$. Since the final payload is subject to aerodynamic forces during its coast to the target, its 
reference area $S_{N+1}$ and fineness ratio $\lambda_{N+1}$ are needed. This explains $i=N+1$ in Eq. (9). In our computations we have chosen $\lambda_{N+1}=5$.

\section{Optimization Problem}

In this section we outline the general features of the optimal interception problem and show by some examples the character of the optimal trajectories. The specified interception data are the flight time of the interceptor $t_{f}$, the target range angle $\theta_{f}$, the target altitude $h_{f}$, and the payload mass $m_{N}^{P}$. The optimization objective is to minimize the total launch mass $m_{0}^{P}$. The free variables in the interception are the angle-of-attack program, the coasting and thrusting periods $t_{i}^{C}$ and $t_{i}^{B}$, and the mass ratio parameters $\sigma_{i}$.

Once the free variables are given it is clear how the corresponding multistage trajectory is generated. Let $r_{0}=$ Earth's radius. Then the multistage nondimensional equations of motion can be written. For the first stage, $R(0)=1, \theta(0)=0$, $\gamma(0)=90 \mathrm{deg}, V(0)=0$, and $M(0)=M_{0}^{P}$. Since the free variables determine $A(\tau)=A_{1}$ (through $M_{1}^{F}$ and $\tau_{1}^{B}$ ) and $M_{0}^{P}$, the equations of motion can be integrated for $0 \leq \tau \leq \tau_{1}^{B}$. At $\tau=\tau_{1}^{B}$, staging occurs and the equations of motion use data appropriate to the second stage. Specifically, Eqs. (1) are integrated for $\tau_{1}^{B} \leq \tau \leq \tau_{1}^{B}+\tau_{2}^{C}+\tau_{2}^{B}$ with $R, \theta, \gamma$, and $V$

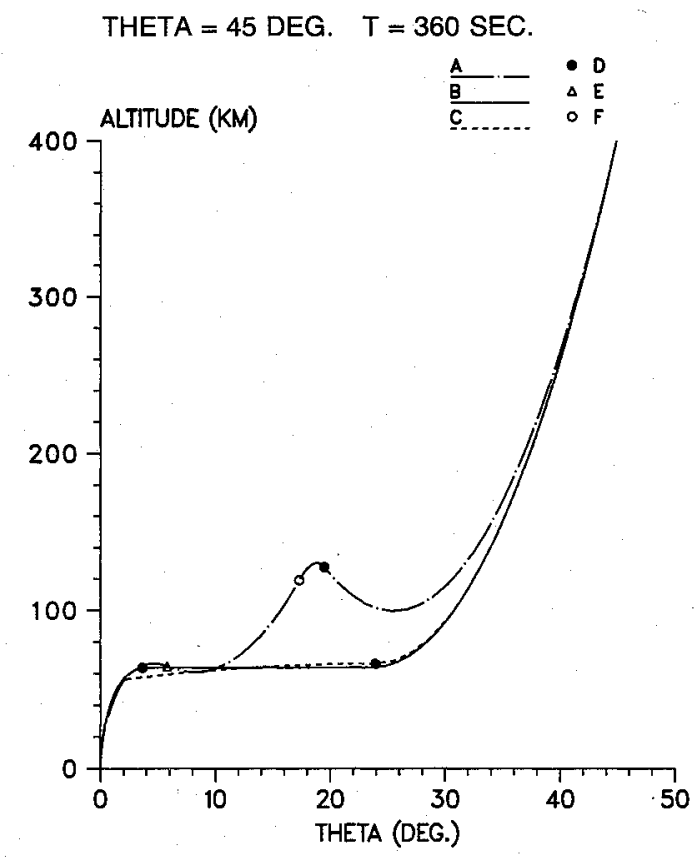

Fig. 1 Optimal trajectories for minimum launch mass: A, Keplerian; B, aeroassisted; C, zero-lift atmospheric; D, burnout of fifth stage; $E$, burnout of fourth stage; F, end of coast for fifth stage. Payload = $10 \mathrm{~kg}$; range angle $=45 \mathrm{deg}$; target altitude $=\mathbf{4 0 0} \mathrm{km}$; flight time $=360 \mathrm{~s}$. continuoùs across staging and with $A(\tau)$ determined by $A_{2}$, $\tau_{2}^{C}$, and $\tau_{2}^{B}$ in the obvious way. The initial mass of the second stage is $M\left(\tau_{1}^{B}\right)=M_{1}^{P}$. The remaining stages are handled in the same way until $\tau=\tau_{f}=\tau_{1}^{B}+\tau_{2}^{C}+\tau_{2}^{B}+\cdots+\tau_{N}^{B}+\tau_{N+1}^{C}$. Actually, this equation is used to determine $\tau_{N+1}^{C}$.

Of course, there are constraints on the free parameters:

$$
0<\sigma_{1}<1, \quad 0<\bar{\tau}_{i}^{B} \leq \tau_{i}^{B}, \quad: \quad 0 \leq \tau_{i}^{C}, \quad i=1, \ldots . N
$$

The lower bounds, $\bar{\tau}_{i}^{B}$, provide a means for limiting the maximum thrust or acceleration of each stage; they may, for instance, depend on other parameters such as $M_{i}^{F}$. In addition to these direct parameter constraints, there are the implicit constraints corresponding to target interception: $\theta\left(\tau_{f}\right)=$ $\theta_{f}, r\left(\tau_{f}\right)=r_{f}$

Operational considerations may add further constraints to the interception problem. For example, we have considered three distinct optimization problems. They differ only in the assumptions placed on the motion of the unpowered final stage; the models for the powered ascent stages remain the same. In the first problem the final payload is constrained to move essentially outside the atmosphere. Thus, the trajectory for the payload is computed easily as a Keplerian transfer from the burnout of stage $N$ to target interception. The constraint is imposed by requiring the minimum altitude of the Keplerian trajectory to exceed a specified altitude. In the second problem, the payload is allowed to move through the atmosphere, but it generates no aerodynamic lift: $\alpha(\tau)=$ $0, \tau_{f}-\tau_{N}^{C} \leq \tau \leq \tau_{f}$. This avoids any increase in drag and heating that may result from lift. In the third problem there is no constraint on the payload so that an aeroassisted coasting trajectory is possible.

Figure 1 and Table 1 show typical numerical results for the three problems. The intercept conditions are $\tau_{f}=360 \mathrm{~s}$, $\theta_{f}=45 \mathrm{deg}$ (approximately $3100 \mathrm{miles}$ ), $h_{f}=400 \mathrm{~km}$ (approximately $250 \mathrm{miles}$ ), payload mass $=m_{N}^{P}=10 \mathrm{~kg}, i_{\mathrm{sp}}=300 \mathrm{~s}$, and $N=5$. In general, the optimal coasting periods for the powered stages turn out to be zero. The only exception is the fifth stage of the Keplerian case.

The launch mass is least for the aeroassisted case. There is a simple physical explanation. The short flight time demands hyperbolic speed $(V>\sqrt{2})$ while simultaneously the trajectory must be kept close to the Earth in order to intercept the target. To resolve these conflicting demands, a net downforce on the vehicle is needed. This force is supplied, without expensive engine thrusting, by negative aerodynamic lift as the payload begins its coast toward the target.

For the zero-lift atmospheric case negative aerodynamic lift is also exploited, but because of the no-lift constraint on the payload it occurs together with engine thrust in the fifth stage. This accounts for the long burn time of this stage; it allows more time for the negative aerodynamic lift to act.

In the Keplerian case the altitude of the payload is constrained to exceed $100 \mathrm{~km}$. This leads to a very large mass

Table 1 Numerical data for optimal trajectories in Fig. 1

\begin{tabular}{|c|c|c|c|c|c|c|}
\hline \multirow{2}{*}{$\begin{array}{l}\text { Type of } \\
\text { solution }\end{array}$} & \multirow{2}{*}{$\begin{array}{l}\text { Launch } \\
\text { mass, kg }\end{array}$} & \multicolumn{5}{|c|}{ Burnout conditions for 4 th stage } \\
\hline & & $h, \mathrm{~km}$ & $V$ & $\theta, \operatorname{deg}$ & $\gamma$, deg & $t, \mathrm{~s}$ \\
\hline Aeroassisted & 18,990 & 58.3 & 1.68 & 2.2 & 3.2 & 40.6 \\
\hline Zero lift atmospheric & 23,070 & 57.0 & 1.89 & 2.5 & 3.5 & 42.5 \\
\hline Keplerian & 50,720 & 64.6 & 1.82 & 5.8 & -1.6 & 75.2 \\
\hline \multirow{2}{*}{$\begin{array}{l}\text { Type of } \\
\text { Solution }\end{array}$} & \multirow{2}{*}{$\begin{array}{l}\text { Coasting time } \\
\text { between } 4 \text { th } \\
\text { and } 5 \text { th stage }\end{array}$} & & \multicolumn{4}{|c|}{ Burnout conditions for 5 th stage } \\
\hline & & $h, \mathrm{~km}$ & $V$ & $\theta, \operatorname{deg}$ & $\gamma, \operatorname{deg}$ & $t, \mathrm{~s}$ \\
\hline Aeroassisted & 0 & 63.7 & 2.11 & 3.7 & 0 & 52.6 \\
\hline Zero lift atmospheric & 0 & 66.5 & 1.96 & 24.0 & 0.005 & 201.9 \\
\hline Keplerian & 90.6 & 127.8 & 2.10 & 19.5 & -4.7 & 182.0 \\
\hline
\end{tabular}


because it essentially eliminates effective utilization of negative aerodynamic lift. The Keplerian solution does have a potential operational advantage. Since the trajectory is essentially outside the atmosphere, communication with the payload is not blocked by atmospheric ionization.

\section{Midcourse Universal Curve}

The aeroassisted trajectory in Fig. 1 has an obvious midcourse segment on which the final payload coasts for an extended period of time until it begins its rise to the target. On the segment there is a negative aerodynamic lift and the path is nearly horizontal. We have observed such segments on all optimal aeroassisted trajectories where performance requirements are high; i.e., $t_{f}$ is small, $\theta_{f}$ is large, and $h_{f}$ is small. Moreover, they can be modeled accurately by a single universal curve that relates altitude and speed. This unique relationship is obtained by balancing the difference between centrifugal and gravity forces with negative aerodynamic lift and minimizing the drag losses by choosing the angle of attack that maximizes the lift-to-drag ratio. As will be seen, the time-dependent motion of the payload on the universal curve also has a universal character.

Our development begins with some notation and simplifying parametric assumptions. Let $-C_{L}^{*}$ and $C_{D}^{*}$ be the lift and drag coefficients that maximize $-C_{L} / C_{D}$. They are obtained from Eqs. (4) and depend only on the fineness ratio, $\lambda=\lambda_{N+1}$, of the payload. Recall that the reference area of the payload, $S_{N+1}$, is given by Eq. (9) and is therefore determined by $\lambda_{N+1}$ and the mass of the payload $m_{N}^{P}$. A dimensionless coefficient, $C_{L}^{*} \eta / 2$, plays an important role in the equations that we will consider. It is given by

$$
\frac{1}{2} C_{L}^{*} \eta=\frac{1}{2} C_{L}^{*}\left(m_{0}\right)^{-1} \rho\left(r_{0}\right) r_{0} S_{N+1}
$$

Since $C_{L}^{*}$ and $S_{N+1}$ are fixed by $\lambda_{N+1}$ and $m_{N}^{P}, C_{L}^{*} \eta / 2$ depends only on $m_{0}$ and $r_{0}$. For the purpose of this section it is convenient to make the following choices for $m_{0}$ and $r_{0}$ :

$$
m_{0}=m_{N}^{P}, \quad \rho\left(r_{0}\right) r_{0}=\frac{3}{2} m_{N}^{P}\left(C_{L}^{*} S_{N+1}\right)^{-1}
$$

This gives $M(\tau) \equiv 1$ in Eqs. (1) and $\frac{1}{2} C_{L}^{*} \eta=\frac{3}{4}$.

The required balance of forces on the nearly horizontal trajectory is achieved by setting the normal acceleration $V \mathrm{~d} \gamma /$ $\mathrm{d} \tau$ and $\gamma$ equal to zero in Eqs. (1) and $C_{L}=-C_{L}^{*}$ in Eqs. (4). The result is the expression for the universal curve:

$$
V=(1+H)^{-\frac{1}{2}}\left[1-\frac{3}{4}(1+H) P(H)\right]^{-\frac{1}{2}}
$$

The reason for choosing $r_{0}$ by Eq. (12) is now clear: $V$ is determined entirely by $P(H)$, and at the reference altitude $(H=0)$ the dimensionless speed has a nice nominal value $(V=2)$. Now let $P(H)$ be given by Eq. (3). Noting that $\mu \cong 10^{3}$, it is easy to show that $1.3 \leq V \leq 4$ implies $|H| \leq 0.6 \times 10^{-3}$. Since the expected varition of $H$ is so small, Eq. (13) is closely approximated by

$$
V=G(H)=\left(1-\frac{3}{4} e^{-\mu H}\right)^{-\frac{1}{2}}
$$

Even for a nonexponential atmosphere this expression is an excellent representation of Eq. (13) because the exponential approximation of $P(H)$ only needs to be accurate for $|\mu H| \leq 0.6$, or $|h| \leq 0.6 h_{s}$. For the payload described in the previous section, $r_{0}$ corresponds to an altitude of $64.0 \mathrm{~km}$ above the Earth's surface.

Along the universal curve there are drag losses and $V$ must decrease. This in turn causes $H, \theta, \gamma$, and $\alpha$ to depend on $\tau$. To obtain these dependencies we begin by substituting Eq. (14) into Eqs. (1) with $A=0$ :

$$
\begin{aligned}
\frac{\mathrm{d} V}{\mathrm{~d} \tau} & =G^{\prime}(H) \frac{\mathrm{d} H}{\mathrm{~d} \tau}=G^{\prime}(H) \frac{\mathrm{d} R}{\mathrm{~d} \tau} \\
& =G^{\prime}(H) G(H) \sin \gamma=-D-\frac{\sin \gamma}{R^{2}}
\end{aligned}
$$

Here, $G^{\prime}(H)$ is the derivative of $G(H)$ so that

$$
G^{\prime}(H)=-\frac{3}{8} \mu e^{-\mu H}[G(H)]^{3}
$$

From this expression it is not difficult to verify that $V=$ $G(H) \geq 1.3$ implies $G^{\prime}(H) G(H)>0.58 \mu$. Since $\mu \cong 10^{3}, R \cong 1$, and $\gamma$ is small, the identity on the right side of Eq. (15) is closely approximated by

$$
D=-G^{\prime}(H) G(H) \sin \gamma
$$

This is the drag required for motion along the universal curve. By Eqs. (2) and (4), $D$ defines $\alpha$ and thus $C_{L}$. Since the line $\mathrm{C}_{L}=\left(-C_{L}^{*} / C_{D}^{*}\right) C_{D}$ is the tangent to the lift-drag polar at $C_{D}^{*}$, it is a good approximation for it in the neighborhood of $C_{D}^{*}$. This approximation with $D$ determined by Eqs. (16) and (17) gives

$$
\left.L=-\frac{3}{8} \frac{C_{L}^{*}}{C_{D}^{*}} \mu e^{-\mu H}[G H)\right]^{4} \sin \gamma
$$

Substituting Eqs. (14) and (18) into Eqs. (1) yields the equations of motion on the universal curve.

To simplify these equations, it is assumed with very little error that $\sin \gamma \cong \gamma, \cos \gamma \cong 1$, and $R=1+H \cong 1$. Then

$$
\begin{aligned}
& \frac{\mathrm{d} H}{\mathrm{~d} \tau}=\gamma G(H) \\
& \frac{\mathrm{d} \theta}{\mathrm{d} \tau}=G(H) \\
& \frac{\mathrm{d} \gamma}{\mathrm{d} \tau}=-\frac{3}{8} \frac{C_{L}^{*}}{C_{D}^{*}} \mu e^{-\mu H}[G(H)]^{3} \gamma+G(H)-[G(H)]^{-1}
\end{aligned}
$$

Because $M(\tau)=1$ and $V(\tau)=G(H(\tau))$ the order of the original system of equations, Eqs. (1), has been reduced from five to three. However, Eqs. (19) are still complicated because they are coupled and nonlinear. Fortunately, the parameter $\mu$ is large, and this leads to a singularly perturbed system whose solution can be obtained analytically.

The nature of the singularly perturbed problem is revealed more clearly by introducing the scaled variables:

$$
\vec{H}=\mu H, \quad \bar{\gamma}=\mu \gamma, \quad \text { and } \quad \bar{G}(\bar{H})=\left(1-\frac{3}{4} e^{-A}\right)^{-\frac{1}{2}}
$$

Then Eqs. (19) become

$$
\begin{aligned}
\frac{\mathrm{d} \bar{H}}{\mathrm{~d} \tau} & =\bar{\gamma} \bar{G}(\bar{H}) \\
\frac{\mathrm{d} \theta}{\mathrm{d} \tau} & =\bar{G}(\bar{H}) \\
\frac{\mathrm{d} \bar{\gamma}}{\mathrm{d} \tau} & =\mu\left\{-\frac{3}{8} \frac{C_{L}^{*}}{C_{D}^{*}} e^{-A}[\bar{G}(\bar{H})]^{3} \bar{\gamma}+\bar{G}(\bar{H})-[\bar{G}(\bar{H})]^{-1}\right\}
\end{aligned}
$$

Standard singular perturbation theory can be applied to this system, ${ }^{3}$ and it shows that the term in the braces goes to zero quickly and that the remaining asymptotic solution satisfies

$$
\frac{\mathrm{d} \bar{H}_{a}}{\mathrm{~d} \tau}=\gamma_{a} \bar{G}\left(\bar{H}_{a}\right)
$$




$$
\bar{\gamma}_{a}=\frac{8}{3} \frac{C_{D}^{*}}{C_{L}^{*}} e^{B_{a}}\left\{\left[\bar{G}\left(\bar{H}_{a}\right)\right]^{-2}-\left[\bar{G}\left(\bar{H}_{a}\right)\right]^{-4}\right\}
$$

This is a first-order system that can be integrated easily when expressed in terms of the variable $y=\left[\bar{G}\left(\bar{H}_{a}\right)\right]^{-1}$.

The details of the integration and backsubstitution to the original problem variables are lengthy and are omitted. They produce the following formulas for the asymptotic motion along the universal curve:

$$
\begin{aligned}
H(\tau) & =\frac{1}{\mu} \hat{H}(x) \\
\theta(\tau)-\theta\left(\tau_{0}\right) & =\frac{C_{L}^{*}}{2 C_{D}^{*}}\left[\hat{\theta}(x)-\hat{\theta}\left(x_{0}\right)\right] \\
\gamma(\tau) & =\frac{2 C_{D}^{*}}{C_{L}^{*} \mu} \hat{\gamma}(x) \\
V(\tau) & =\hat{V}(x) \\
x & =x_{0}+\frac{2 C_{D}^{*}}{C_{L}^{*}}\left(\tau-\tau_{0}\right)
\end{aligned}
$$

where

$$
\begin{aligned}
\hat{V}(x) & =\left(3 e^{x}-1\right)^{-1}\left(3 e^{x}+1\right) \\
\hat{\gamma}(x) & =[\hat{V}(x)]^{-2} \\
\hat{H}(x) & =-\ell x_{3}^{4}[1-\hat{\gamma}(x)] \\
\theta(x) & =-x+2 \ell x_{\frac{1}{2}}\left(3 e^{x}-1\right)
\end{aligned}
$$

and $x_{0}$ is determined by $\hat{V}\left(x_{0}\right)=V\left(\tau_{0}\right)$, or, equivalently, by

$$
x_{0}=\ln \frac{1}{3}\left[V\left(\tau_{0}\right)-1\right]^{-1}\left[V\left(\tau_{0}\right)+1\right]
$$

It is easy to confirm that the preceding formulas cause $H$ and $V$ to lie on the universal curve. Also, using Eqs. (2), (14), (16), (17), and (24), it follows that

$$
\begin{aligned}
C_{D} & =D\left(\frac{1}{2} \eta e^{-\mu H} V^{2}\right)^{-1} \\
& \cong-G^{\prime}(H) G(H) \gamma\left(\frac{1}{2} \eta e^{-\mu H} V^{2}\right)^{-1}=C_{D}^{*}
\end{aligned}
$$

Thus, within the accuracy of the approximation $\sin \gamma \cong \gamma$, the motion of the universal curve actually does maximize the lift-to-drag ratio.

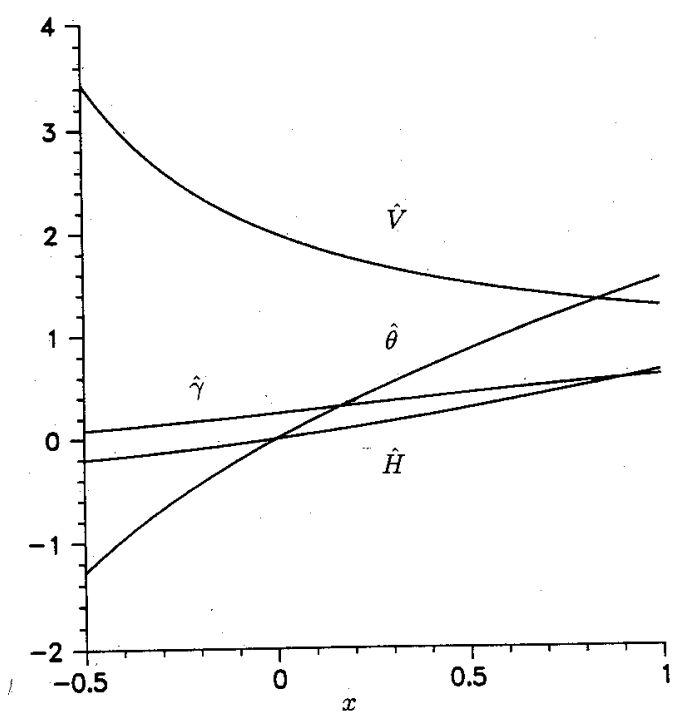

Fig. 2 Functions describing motion on the universal curve.
The functions $\hat{H}, \hat{\theta}, \hat{\gamma}$, and $\hat{V}$ are plotted in Fig. 2. The ranges of variables that are shown are adequate for any reasonable target conditions. For example, with $C_{L}^{*} /$ $C_{D}^{*}=2.5$, the value corresponding to $\lambda=5$, a unit change in $\hat{\theta}$ corresponds to a $72-\mathrm{deg}$ change in $\theta$. For the entire range that is shown, $|\hat{\gamma}| \leq 0.61$. This implies $|\gamma| \leq 0.61 \mu^{-1} \cong 6 \times$ $10^{-4} \mathrm{rad}=0.034 \mathrm{deg}$. The departure of $\gamma$ from zero is indeed small.

\section{Numerical Optimization Procedure}

\section{A. General Discussion}

Over the years many procedures have been developed for the numerical solution of problems in optimal control. One approach is to write the necessary conditions and solve the resulting two-point boundary-value problem by well-developed techniques. ${ }^{4,5}$ There are difficulties in applying this approach to the interceptor problem. The two-point boundary-value problem is replaced by a complex multipoint boundary-value problem, and derivatives of the nonlinear functions appearing in the problem statement are needed. Moreover, reasonably good initial estimates of the optimal solution and the corresponding necessary condition multipliers are needed. Various gradient methods ${ }^{6,7}$ can be extended with less difficulty to the multistage problem, but at best convergence is slow and terminal conditions cannot be met accurately. Second-order gradient methods ${ }^{8,9}$. have better convergence rates, but they require second derivatives of the problem functions that are difficult to obtain because of the complex nonlinear and multistage character of the interceptor problem.

In view of the aforementioned difficulties it seems most appropriate to use a direct method in which the infinitedimensional optimal control problem is replaced by a finitedimensional approximation. Many approaches for doing this have appeared in the literature (see, for example, the review given by Hargraves and Paris ${ }^{10}$ ). In our approach the angleof-attack program $\alpha(\tau)$ is approximated by a finite-dimensional functional parameterization, and the equations of motion with staging conditions are integrated accurately. The details of the overall process are described in the following subsections. Other direct methods, such as collocation methods, ${ }^{10}$ could also be applied to the interceptor problem. A possible advantage would be the elimination of special procedures, such as the one described in the next subsection, for improving numerical conditioning. Collocation methods, as well as many other direct methods, do have a potential disadvantage. An accurate solution of the differential equations may necessitate a fine collocation grid, which in turn increases the dimension of the finite-dimensional optimization problem.

\section{B. Indirect Parameterization of $\alpha(\tau)$}

In the approximation of the angle-of-attack program several questions arise: the dimension and the form of the functional representation, the accuracy of the corresponding approximate optimum solution, and the conditioning of the resulting numerical optimization problem. Our experience shows that relatively low-dimensional parameterizations are quite effective, provided the approximating functions are chosen with some care. The dimensionality is important because it has a strong effect on the computational time. Conditioning affects both the speed and reliability of the optimization process.

A direct parameterization of $\alpha(\tau)$ is the most obvious way to proceed. For example, a continuous piecewise linear function, which is parameterized by its values at its joints (points of slope discontinuity), is simple and has flexibility in that the joints may be placed closely where rapid changes in $\alpha(\tau)$ are expected. The fatal shortcoming of direct parameterizations is poor conditioning of the optimization problem. The terminal constraints are very sensitive with respect to small changes in $\alpha(\tau)$, especially when the changes occur early in the flight. This 
is not surprising in view of the open-ended integrations that occur in solving the equations of motion.

To circumvent this poor conditioning, it is better to parameterize $\alpha(\tau)$ indirectly through a direct parameterization of $\gamma(\tau)$. In this approach $\mathrm{d} \gamma / \mathrm{d} \tau$ is computed from the parameterized $\gamma(\tau)$ and substituted, together with $R, \gamma, V$, and $M$, into Eq. (1c). This equation then becomes through $\sin \alpha$ and $L$ an implicit equation in $\alpha$. The indirect parameterization of $\alpha$ is obtained by solving the implicit equation. Note that this eliminates the need to integrate the differential equation for $\mathrm{d} \gamma / \mathrm{d} \tau$. Of course, the remaining differential equations in Eqs. (1) must be integrated as usual. When the indirect parameterization is used, the entire trajectory is under more direct control. Changes in $\gamma(\tau)$ near the beginning of the trajectory have very little effect on the terminal portions of the trajectory. Thus, the sensitivity of the terminal constraints to the parameterization is greatly reduced. Another advantage, perhaps less important, is evident at launch. Here, the differential equation for $\mathrm{d} \gamma / \mathrm{d} \tau$ has a singularity because $V=0$. With the $\gamma$ parameterization the differential equation is not integrated and the normally troublesome singularity is circumvented.

The implicit equation for $\alpha$ presents possible difficulties. An inadmissible $\gamma(\tau)$ may be specified, i.e., one in which the implicit equation has no solution. This corresponds physically to requiring more transverse acceleration than is available. In most of our work inadmissibility has not been a problem, provided the line search in the optimization algorithm has a procedure for reducing step size when it produces an inadmissible $\gamma(\tau)$. Occasionally, when $\alpha$ must be very large, as in the fifth stage of the Keplerian problem of Fig. 1, it is better to parameterize $\alpha(\tau)$ directly, keeping the $\gamma$ parameterization for the other stages. The actual numerical solution of the implicit equation is straightforward. One approach is to use several Newton iterations. Alternatively, $L$ may be approximated by a quadratic function of $\sin \alpha$; then the implicit equation is quadratic in $\sin \alpha$ and may be solved by a formula.

Another issue is the smoothness of $\alpha(\tau)$ and other problem variables. If $\gamma(\tau)$ is continuous but has slope discontinuities, as in the case of a piecewise linear parameterization, the differentiation of $\gamma(\tau)$ causes $\alpha(\tau)$ to be discontinuous. Even for smoother parameterizations the problem is not avoided. At the staging times the vehicle mass, thrust, and aerodynamic parameters change discontinuously, and this causes discontinuities in $\alpha(\tau)$. Continuity of $\alpha(\tau)$ can be imposed by introducing, through the functional representation of $\gamma(\tau)$, an appropriate jump in $\mathrm{d} \gamma / \mathrm{d} \tau$ at the staging time. The value of $\mathrm{d} \gamma / \mathrm{d} \tau$ just after staging, $(\mathrm{d} \gamma / \mathrm{d} \tau)^{+}$; is evaluated by using its defining equation [Eq. (1c)] with $R, \gamma, V$, and $\alpha$ continuous across staging and the changes in $M, A$, and aerodynamic parameters determined by the staging equations of Sec. II.

It is also possible to introduce other smoothness constraints. For example, consider the pitch angle $\psi=\alpha+\gamma$. Although $\psi$ does not appear in the point mass equations, it has practical implications because the moment applied to the vehicle is proportional to its second derivative. To avoid an impulsive moment it is necessary to require continuity of $\mathrm{d} \psi / \mathrm{d} \tau$. This leads to the condition $(\mathrm{d} \alpha / \mathrm{d} \tau)^{+}-(\mathrm{d} \alpha /$ $\mathrm{d} \tau)^{-}=(\mathrm{d} \gamma / \mathrm{d} \tau)^{+}-(\mathrm{d} \gamma / \mathrm{d} \tau)^{-}$, where the superscripts give the values immediately before and after staging. In order for the derivatives with respect to $\alpha$ to exist, it is certainly necessary that $\alpha(\tau)$ be continuous. Thus, as in the previous paragraph, both $(\mathrm{d} \gamma / \mathrm{d} \tau)^{+}$and $(\mathrm{d} \gamma / \mathrm{d} \tau)^{-}$are known. The resulting condition on $(\mathrm{d} \alpha / \mathrm{d} \tau)^{+}-(\mathrm{d} \alpha / \mathrm{d} \tau)$ can be obtained by differentiating the $\mathrm{d} \gamma / \mathrm{d} \tau$ equation once. This in turn defines the value of $\left(d^{2} \gamma / d \tau^{2}\right)^{+}$. Thus, conditions on both $(d \gamma / d \tau)^{+}$and $\left(d^{2} \tau /\right.$ $\left.\mathrm{d} \tau^{2}\right)^{+}$are obtained.

Our numerical experience has produced some guidelines on the functional form of the $\gamma$ parameterization. Because of the large changes in $\gamma$ encountered in the first stage, it has been found necessary to use a parameterization with at least three free parameters. Since $\gamma(0)=90 \mathrm{deg}$, a cubic in $\tau$ satisfies this requirement and has been as effective as any other choice. For the remaining stages one free parameter suffices. When there are no smoothness constraints, a linear function works well. Only one parameter is involved for each stage because $\gamma(\tau)$ must be continuous across staging. When the various continuity constraints are imposed, a more elaborate, one-parameter representation is needed. For instance, when $\mathrm{d} \psi / \mathrm{d} \tau$ is continuous, we have found it effective to use $\gamma(\tau)=a+b \tau+$ $c e^{-\sigma \tau}+d e^{-v \tau}$. The parameters $c$ and $d$ allow the matching of the constraints on $(\mathrm{d} \gamma / \mathrm{d} \tau)^{+}$and $\left(\mathrm{d}^{2} \gamma / \mathrm{d} \tau^{2}\right)^{+}$. The values $\sigma$ and $v$ are chosen so that the effect of the continuity conditions does not persist for too long. For a given functional form the free parameters may be defined in different ways. It has been found that problem conditioning is usually better if values of $\gamma$ at specified interpolation points are used. For example, when there is one free parameter for a stage, the value of $\gamma$ at the thrust termination time is a good choice.

\section{Formulation of the Finite-Dimensional Problem}

The variables in the finite-dimensional optimization problem are the parameters in the representation of $\gamma(\tau)$ and the staging parameters: $\sigma_{i}, \tau_{i}^{B}$, and $\tau_{i}^{C}$, for $i=1, \ldots, N$. The constraints on these parameters [Eq. (10)] are implemented by means of nonlinear transformations:

$$
\begin{aligned}
\sigma_{i} & =(1-\varepsilon)\left(0.5+0.5 \sin \phi_{i}\right) \\
\tau_{i}^{B} & =0.5\left(\bar{\tau}_{i}^{B}+\tilde{\tau}_{i}^{B}\right)+0.5\left(\bar{\tau}_{i}^{B}-\tilde{\tau}_{i}^{B}\right) \sin v_{i} \\
\tau_{i}^{C} & =\tilde{\tau}_{i}^{C}\left(0.5+0.5 \sin \mu_{i}\right)
\end{aligned}
$$

In Eq. (27) $\varepsilon>0$ is a small positive number that implements the strict inequalities on $\sigma_{i}$. The variables $\phi_{i}, v_{i}$, and $\mu_{i}$ become unconstrained variables in the finite-dimensional optimization problem. The parameters $\tilde{\tau}_{i}^{B}$ and $\tilde{\tau}_{i}^{C}$ are scaling parameters that establish upper limits for $\tau_{i}^{B}$ and $\tau_{i}^{C}$ and improve the numerical conditioning of the optimization problem. The nonlinearities introduced by the transformations do not appear to affect the speed or reliability of the numerical optimization process. If coasting of the powered stages is not allowed and the guidelines of the preceding subsection are followed, there is a total of $3 N+2$ variables: $N$ each for Eqs. (27) and (28), three for the parameterization of $\gamma$ in the first stage, and one for the parameterization of $\gamma$ in each of the remaining stages.

The remaining constraints in the optimization problem are the terminal conditions $\theta\left(\tau_{f}\right)=\theta_{f}$ and $R\left(\tau_{f}\right)=R_{f}$. Errors in meeting these equality conditions are evaluated by integrating the differential equations of motion, which are fully defined once the variables of the preceding paragraph are specified. The cost to be minimized is $M_{0}^{P}$, obtained from the $\sigma_{i}$ by Eq. (6).

\section{Numerical Evaluations}

In order to implement the optimization algorithm it is necessary to numerically evaluate $\theta\left(\tau_{f}\right), R\left(\tau_{f}\right)$, and $M_{0}^{P}$ and their gradients with respect to the variables. To avoid the very complex direct evaluation of the gradients, first-order finite differences on $\theta\left(\tau_{f}\right), R\left(\tau_{f}\right)$, and $M_{0}^{P}$ are used. This is numerically expensive because each evaluation of the gradient requires $n+1$ integrations of the differential equations, where $n$ is the number of variables. Techniques for speeding the integrations have been reported by Howe et al. ${ }^{11}$ For the specific results reported here a fixed-step RK-4 procedure was used with approximately 10 steps per stage. Rounding and truncation errors in the computation of the gradients can seriously degrade the performance of the minimization algorithm. In this regard the naturally good scaling of the dimensionless equations [Eqs. (1)] is a distinct advantage. Balancing the rounding and truncation errors by a proper choice of the finite-difference increment is also important. ${ }^{12}$

For cases where the payload stage closely follows the universal curve, it is possible to eliminate for the payload the 
parameterization of $\gamma(\tau)$ and the numerical integration of the equations of motion. At the time of final-stage burnout, $V\left(\tau_{b}\right)$ is known. By setting $\tau_{0}=\tau_{b}$, formulas $(23-25)$ define the
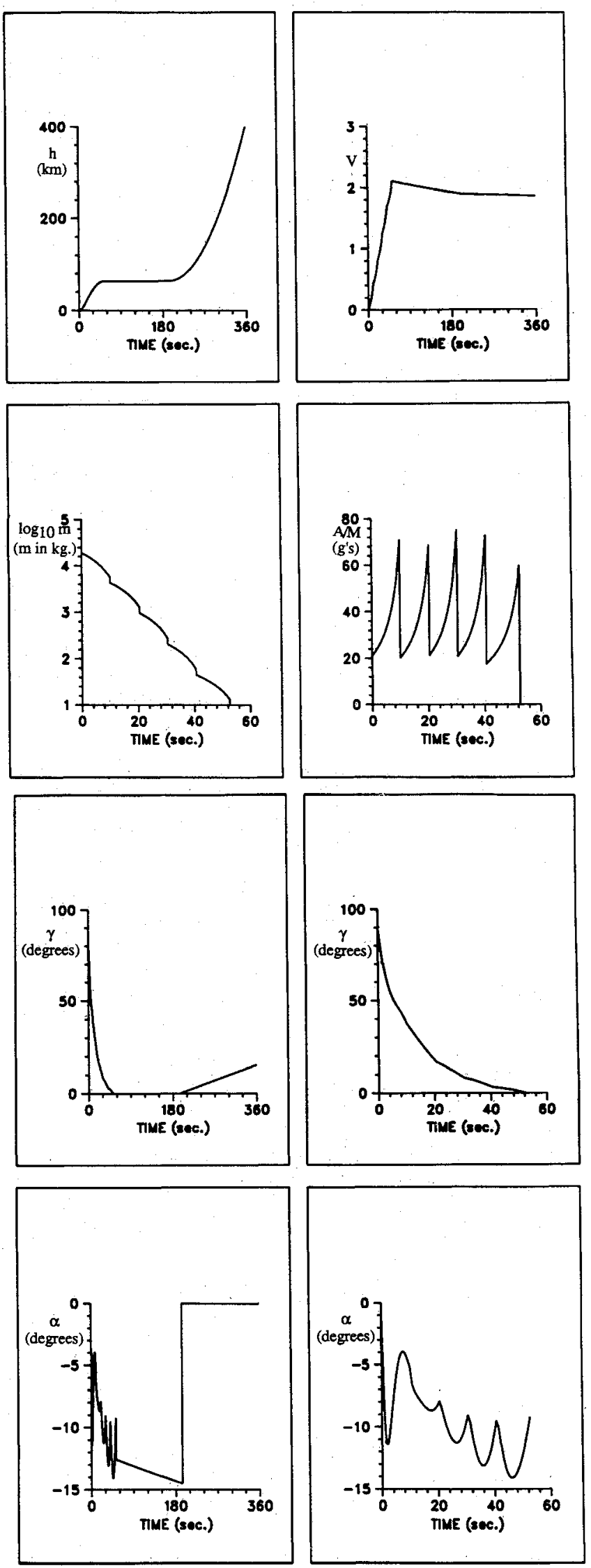

Fig. 3 Additional details of the optimal aeroassisted trajectory shown in Fig. 1. motion along the universal curve. In general the conditions $V\left(\tau_{b}\right)=G\left[H\left(\tau_{b}\right)\right]$ and $\gamma\left(\tau_{b}\right)=0$ (a very accurate approximation since $|\gamma| \leq 6 \times 10^{-4}$ on the universal curve) are not satisfied by the ascent trajectory; thus, they must be imposed as additional equality constraints. The universal curve is followed until a time $\tau=\tau_{d}$ is reached. After this time $\alpha(\tau)$ is set to zero. Because there is no aerodynamic lift and $V>1$, the trajectory then departs from the universal curve and rises toward the target. By evaluating this departure trajectory at $\tau=\tau_{f}$, the errors in the target conditions are determined. Because $\gamma\left(\tau_{b}\right)=0$, one variable is omitted from the parameterization of $\gamma(\tau)$; this is balanced by the addition of the parameter $\tau_{d}$.

\section{E. Optimization Algorithm}

The equality constraints are treated by the augmented Lagrangian method, which reduces the constrained problem to a sequence of unconstrained problems. Both the penalty coefficients and the multipliers are updated automatically by a scheme that is described on page 292 of Fletcher. ${ }^{13}$ The unconstrained minimization program used in our computations is a variant of the BFGS, quasi-Newton implementation due to Shanno and Phua. ${ }^{14}$ It provides superlinear convergence without requiring the evaluation of second derivatives. A reset procedure has been added that greatly reduces the probability that the descent will terminate prematurely due to accumulation of round-off errors in the quasi-Newton update.

Generally, the performance of the overall optimization procedure has been very satisfactory. The number of gradient evaluations required to obtain a solution is between 300 and 400. This is not unreasonable for the typical number of problem variables: 17 for $\dot{N}=5$ and 20 for $N=6$. Errors in meeting the specified target conditions are very small: about $1 \mathrm{~m}$ in altitude and about $0.05 \mathrm{deg}$ in range angle. The computational time for a solution is typically between 60 and $90 \mathrm{~min}$ on an Apollo DN 4000.

\section{Some Specific Results}

Many optimal intercept problems have been solved using the methods and models of the preceding sections. In this section we summarize some of our results for the aeroassisted problem. The pitch angle is constrained to have a continuous derivative except at the entry and exit points of the universal curve, where $\alpha(\tau)$ is allowed to be discontinuous. A variety of problems have been solved where coasting of the stages is allowed. In these problems the optimal coasting times are

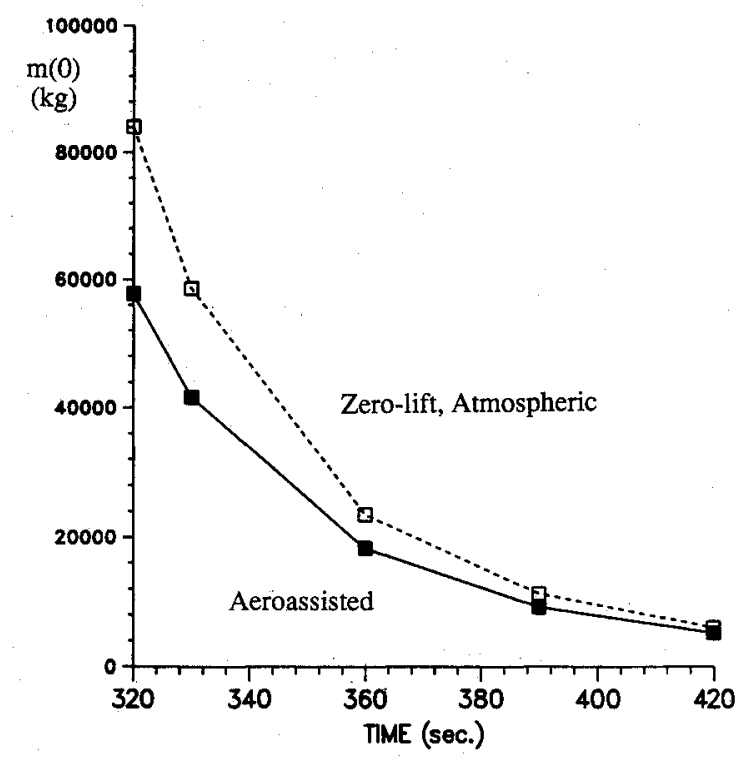

Fig. 4 Optimal launch mass as a function of flight time. Number of stages $=5$; payload $=10 \mathrm{~kg}$; range angle $=45 \mathrm{deg}$; target altitude $=$ $200 \mathrm{~km}$; specific impulse $=300 \mathrm{~s}$. 
either zero or so small that they have little effect on the optimal launch mass. For all the problems considered here the coasting times have been set to zero. The minimum burn time for each stage is $10 \mathrm{~s}$, and the payload mass is $10 \mathrm{~kg}$. Except for the results in Fig. 6, the specific impulse is fixed at 300 s.

Additional details of the optimal aeroassisted solution described in Sec. III are shown in Fig. 3. The staging times are apparent. With the exception of the fifth stage, the optimal burn times are at their lower limits of $10 \mathrm{~s}$. The resulting vehicle acceleration $A / M$ is quite high, ranging from about 20 to $70 \mathrm{~g}$. If the minimum burn time is reduced below $10 \mathrm{~s}$, even higher accelerations are obtained. The universal curve is followed from about $53 \mathrm{~s}$ to $194 \mathrm{~s}$. The slight drop in $V$ predicted by Fig. 2 is evident. There is little loss in speed after the exit from the universal curve. The reason is obvious from Fig. 1: The trajectory leaves the atmosphere quickly; hence, there is little aerodynamic drag. Close inspection of $\gamma(\tau)$ for $0<\tau<60$ shows its linear-exponential parameterization. If the smoothness constraint on the pitch angle is removed and $\alpha(\tau)$ is allowed to be discontinuous at the staging points, the

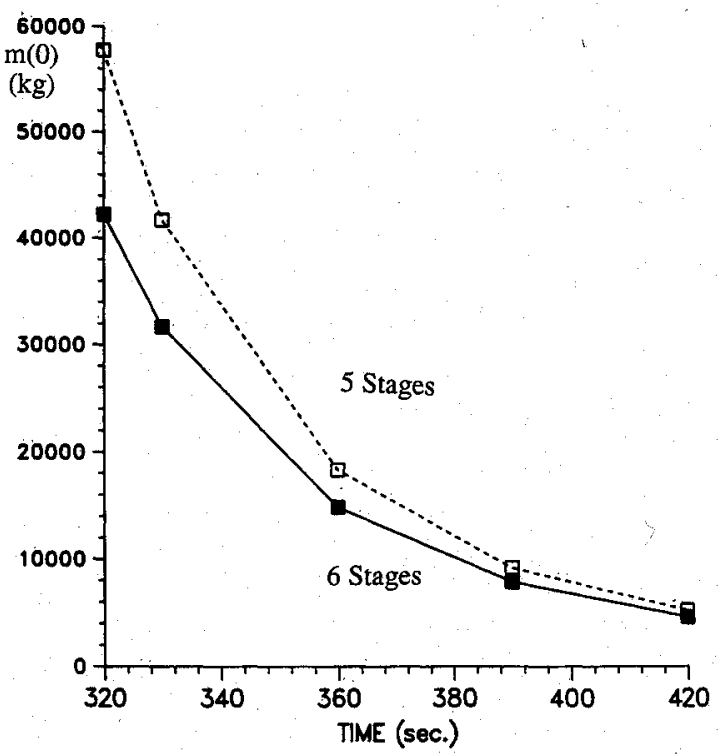

Fig. 5 Optimal launch mass as a function of flight time for aeroassisted trajectories. Payload $=10 \mathrm{~kg}$; range angle $=\mathbf{4 5} \mathrm{deg}$; target altitude $=200 \mathrm{~km}$; specific impulse $=300 \mathrm{~s}$.

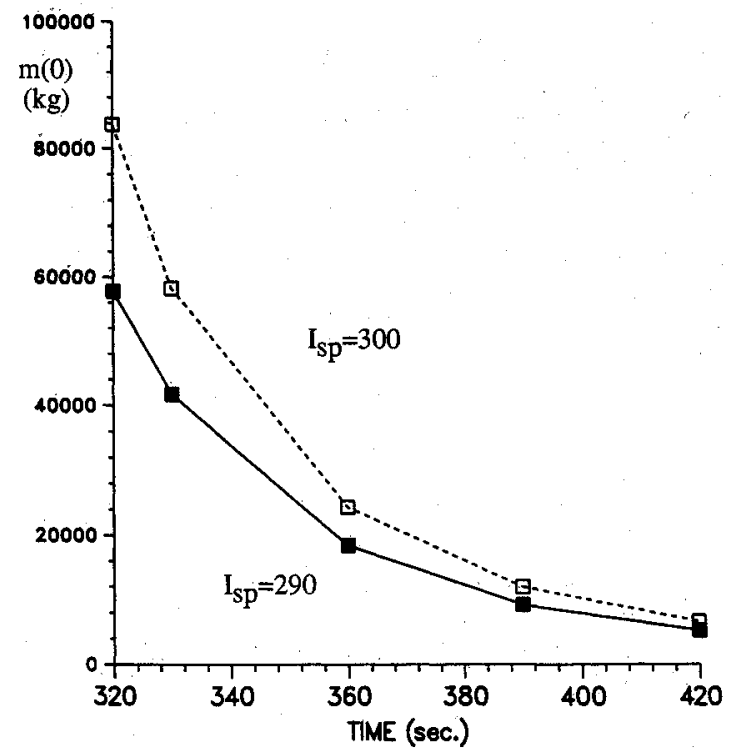

Fig. 6 Optimal launch mass as a function of flight time for aeroassisted trajectories. Number of stages $=5$; payload $=10 \mathrm{~kg}$; range angle $=\mathbf{4 5} \mathrm{deg}$; target altitude $=\mathbf{2 0 0} \mathrm{km}$. overall character of the trajectory is essentially the same and the launch mass is reduced by about $0.5 \%$. Thus, the smoothness of the pitch angle does not seem to be a very stringent constraint.

Figures 4-6 illustrate the effect of key parameters on the optimal launch mass. Note that the target altitude is $200 \mathrm{~km}$, a more demanding intercept condition than the $400 \mathrm{~km}$ of the preceding problem. Figure 4 shows the relative performance of optimal aeroassisted trajectories and optimal zero-lift, atmospheric trajectories. The advantage of aeroassisted trajectories is greater for shorter intercept times. For both types of trajectories the launch mass grows very rapidly as the flight time approaches $320 \mathrm{~s}$. Figure 5 shows the difference between five- and six-stage aeroassisted interceptors. The six-stage interceptor has a decided advantage for shorter flight times. Figure 6 shows the affect of reducing the specific impulse. The $10-\mathrm{s}$ reduction increases the launch mass by over $40 \%$ for the shorter flight times.

Additional solution results for the aeroassisted case suggest other trends. If all other parameters are fixed, the optimal launch mass varies little if the range angle and intercept time of the target vary in direct proportion. Increasing the axial drag coefficient $C_{A}$ by 0.02 increases the launch mass by about $10 \%$. Increasing the mass density of the stages by $50 \%$ decreases the launch mass by about $6 \%$. More accurate models of atmospheric density have very little effect on the launch mass.

\section{Conclusions}

Models for high-performance, multistage rocket interceptors have been introduced. Efficient numerical procedures for minimizing their launch mass with respect to staging parameters and the angle-of-attack program have been described. These procedures circumvent many of the complexities associated with the nonlinear, multistage character of the minimization problem. Even though the angle-of-attack program is represented approximately, the equations of motion are solved accurately. Inherent ill-conditioning of the procedure is overcome by parameterizing the control variable (angle of attack) indirectly by a direct parameterization of a state variable (flight-path angle). A key aspect of the optimal trajectories is the negative-lift, aeroassisted character of the coasting payload stage. A simple and accurate analytical model of this coasting motion (the universal curve) has been derived. This model may prove useful in other problems where planetary atmospheres are encountered at hyperbolic speeds. The effects of various design parameters and target conditions on the optimal launch mass are described. As the intercept time is reduced, the optimal launch mass rises at an ever-increasing rate.

\section{Acknowledgments}

The research reported in this paper has been supported by the U.S. Army Strategic Defense Command Contract DASG60-88-C-0037. The authors thank David Parag for his helpful contributions.

\section{References}

${ }^{1}$ Vinh, N. X., Buseman, A., and Culp, R. D., Hypersonic and Planetary Entry Flight Mechanics, Univ. of Michigan Press, Ann Arbor, MI, 1980.

${ }^{2}$ Jorgensen, L. H., "Estimation of Aerodynamics for Slender Bodies Alone and with Lifting Surfaces," AIAA Journal, Vol. 11, No. 3, 1975, pp. 409-412.

${ }^{3} \mathrm{O}$ 'Malley, R. E., Jr., "On Nonlinear Singularly Perturbed Initial Value Problems," SIAM Review, Vol. 30., No. 2, 1988, pp. 193-212.

${ }^{4}$ Keller, H. B., Numerical Solution of Two Point Boundary Value Problems, Society for Industrial and Applied Mathematics, Philadelphia, PA, 1976.

${ }^{5}$ Bulirsch, R., and Stoer, J., Introduction to Numerical Analysis, Springer-Verlag, New York, 1980.

${ }^{6} \mathrm{Kelly}, \mathrm{H}$. J., "Method of Gradients," Optimization Techniques, edited by G. Leitmann, Academic, New York, 1962, pp. 205-254. 
${ }^{7}$ Bryson, A. E., Jr., and Ho, Y. C., Applied Optimal Control, Halsted, Washington, DC, 1975.

${ }^{8}$ Kelly, H. J., Kopp, R. E., and Moyer, H. J., "A Trajectory Optimization Technique Based on the Theory of Second Variation," Progress in Astronautics and Aeronautics, Vol. 14, Academic, New York, 1964, pp. 559-582.

${ }^{9}$ Miele, A., Mangiavacchi, A., and Agarwal, A. K., "Modified Quasilinearization Algorithm for Optimal Control Problems with Nondifferential Constraints," Journal of Optimization Theory and Applications, Vol. 14, No. 5, 1974, pp. 529-556.

${ }^{10}$ Hargraves, C. R., and Paris, S. W., "Direct Trajectory Optimization Using Nonlinear Programming and Collocation," Journal of
Guidance, Control, and Dynamics, Vol. 10, No. 4, 1987, pp. 338-342.

${ }^{11}$ Howe, R. M., Gilbert, E. G., Lu, P., and Vinh, N. X., "Trajectory Optimization of Earth-Launched Interceptors at Supercircular Speeds," Proceedings of the International Society for Optical Engineering, Vol. 872, 1988, pp. 12-18.

${ }^{12}$ Gill, P. E., Murray, W., and Wright, M. H., Practical Optimization, Academic, New York, 1981.

${ }^{13}$ Fletcher, R., Practical Methods of Optimization, 2nd ed., WileyInterscience, New York, 1987.

${ }^{14}$ Shanno, D. F., and Phua, K. H., "Minimization of Unconstrained Multivariate Functions," ACM Transactions on Mathematical Software, Vol. 6, 1980, pp. 618-622.

\section{Monitoring Earth's Ocean, Land and Atmosphere from Space: Sensors, Systems, and Applications}

\section{Abraham Schnapf, editor}

This comprehensive survey presents previously unpublished material on past, present, and future remote-sensing projects throughout the world. Chapters examine technical and other aspects of seminal satellite projects, such as Tiros/NOAA, NIMBUS, DMS, LANDSAT, Seasat, TOPEX, and GEOSAT, and remote-sensing programs from other countries. The book offers analysis of future NOAA requirements, spaceborne active laser sensors, and multidisciplinary Earth observation from space platforms.

TO ORDER: Write, Phone, or FAX:

c/o TASCO, 9 Jay Gould Ct., P.O. Box 753

Waldorf, MD 20604 Phone (301) 645-5643

Dept. 415 FAX (301) 843-0159

Sales Tax: CA residents, $7 \%$; DC, $6 \%$. Add $\$ 4.50$ for shipping and handling. Orders under $\$ 50.00$ must be prepaid. Foreign orders must be prepaid.

Please allow 4 weeks for delivery. Prices are subject to change without notice. Returns will be accepted within 15 days.
1985830 pp., illus. Hardback

ISBN 0-915928-98-1

AIAA Members $\$ \mathbf{\$ 5 9 . 9 5}$

Nonmembers $\$ 99.95$

Order Number V-97 